\title{
Reconfiguring Triangulations with Edge Flips and Point Moves
}

\author{
Greg Aloupis ${ }^{1}$, Prosenjit Bose ${ }^{2}$, and Pat Morin ${ }^{2}$ \\ 1 School of Computer Science, McGill University \\ athens@cs.mcgill.ca \\ 2 School of Computer Science, Carleton University \\ \{jit,morin\}@scs.carleton.ca
}

\begin{abstract}
We examine reconfigurations between triangulations and near-triangulations of point sets, and give new bounds on the number of point moves and edge flips sufficient for any reconfiguration. We show that with $O(n \log n)$ edge flips and point moves, we can transform any geometric near-triangulation on $n$ points to any other geometric neartriangulation on $n$ possibly different points. This improves the previously known bound of $O\left(n^{2}\right)$ edge flips and point moves.
\end{abstract}

\section{Introduction}

An edge flip is a graph operation that is defined on (near)-triangulations ${ }^{1}$. An edge flip on a triangulation is simply the deletion of an edge, followed by the insertion of another edge such that the resulting graph remains a triangulation. The definition of an edge flip gives rise to several natural questions: Does there always exist a sequence of flips that reconfigures a given triangulation to any other triangulation? Are there bounds on the lengths of such sequences if they exist? Can these sequences be computed? These questions have been studied in the literature in many different settings. In particular, Wagner [19] proved that given any two $n$-vertex triangulations $G_{1}$ and $G_{2}$, there always exists a finite sequence of edge flips that reconfigures $G_{1}$ into a graph isomorphic to $G_{2}$. Subsequently, Komuro [10] showed that in fact $O(n)$ edge flips suffice. Recently, Bose et al. [2] showed that $O(\log n)$ simultaneous edge flips suffice and are sometimes necessary. This setting of the problem is referred to as the combinatorial setting since the triangulations are only embedded combinatorially, i.e. only the cyclic order of edges around each vertex is defined.

In the geometric setting, the graphs are embedded in the plane with edges represented by straight line segments. Pairs of edges can only intersect at their endpoints. Edge flips are still valid operations in this setting, except that now the edge that is added must be a line segment that cannot properly intersect any of the existing edges of the graph. This implies that there are valid edge flips

\footnotetext{
* Research supported in part by the Natural Science and Engineering Council of Canada.

${ }^{1}$ A triangulation is a plane graph where every face is a triangle. In a neartriangulation, the outer face may not be a triangle.
} 
in the combinatorial setting that are no longer valid in the geometric setting. Lawson [12] showed that given any two geometric near-triangulations $N_{1}$ and $N_{2}$ embedded on the same $n$ points in the plane, there always exists a finite sequence of edge flips that transforms the edge set of $N_{1}$ to the edge set of $N_{2}$. Hurtado, Noy and Urrutia [9] showed that $O\left(n^{2}\right)$ flips are always sufficient and that $\Omega\left(n^{2}\right)$ flips are sometimes necessary.

Note that in the geometric setting, only the near-triangulations that are defined on the specified point set can be attained via edge flips. For example, no planar $K_{4}$ can be drawn on a convex set of four points without introducing a crossing.

In order to resolve the discrepancy between the combinatorial and geometric settings, Abellanas et al. [1] introduced a geometric operation called a point move. A point move on a geometric triangulation is simply the modification of the coordinates of one vertex such that after the modification the graph remains a geometric triangulation. That is, the move is valid provided that after moving the vertex to a new position, no edge crossings are introduced. They also showed that with $O\left(n^{2}\right)$ edge flips and $O(n)$ point moves, any geometric triangulation on $n$ points can be transformed to any other geometric triangulation on $n$ possibly different points.

The question which initiated our investigation is whether or not $O\left(n^{2}\right)$ edge flips are necessary. In this paper, we show that with $O(n \log n)$ edge flips and point moves, we can transform any geometric near-triangulation on $n$ points to any other geometric near-triangulation on $n$ possibly different points. Next, we show that if we restrict our attention to geometric near-triangulations defined on a fixed point set of size $n$, the problem is just as difficult even with the use of point moves. Finally, we show that with a slightly more general point move, we can remove the extra log factor from our main result.

\section{Results}

In the remainder of the paper, all triangulations and near-triangulations are geometric. It is assumed that the outer face any given near-triangulation is convex, and that any two near-triangulations involved in a reconfiguration have the same number of points on the convex hull.

We assume that the $n$ vertices of any given triangulation are in general position. It is not difficult to see that $O(n)$ point moves can reconfigure a triangulation to this form. We begin with some basic building blocks that will allow us to prove the main theorems.

Lemma 1. [2] A reconfiguration between two triangulations of the same point set that is in convex position can be done with $O(n)$ edge flips.

Lemma 2. [9] Let $v_{1}, v_{2}$ and $v_{3}$ be three consecutive vertices on the outer face of a near-triangulation $T_{1}$. Let $C$ be the path from $v_{1}$ to $v_{3}$ on the convex hull of all vertices but $v_{2}$. A near-triangulation $T_{2}$ containing all edges of $C$ may be constructed from $T_{1}$ with $t$ edge flips, where $t$ is the number of edges initially intersecting $C$ in $T_{1}$. 
Lemma 3. Given a near-triangulation $T$, any vertex $p \in T$ with degree $d>3$ that is inside the convex hull of the vertices of $T$ can have its degree reduced to 3 with $d-3$ edge flips.

Proof. Let $P$ be the polygon that is the union of all triangles incident to $p$. By Meister's two-ears theorem [13], if $P$ has more than three vertices, then it has at least two disjoint ears ${ }^{2}$. At most one of them can contain $p$. Therefore $p$ and one of the ears form a convex quadrilateral. We may flip the edge from $p$ to the tip of the ear, effectively cutting the ear from $P$ and reducing the number of vertices of $P$ by one. This process may be continued until $P$ is reduced to a triangle that contains $p$ as desired.

Lemma 4. Given a near-triangulation $T$, any vertex $p \in T$ with degree 3 that is inside the convex hull of the vertices of $T$ can be moved to a new position in the triangulation along a straight path crossing $t$ edges, using at most $2 t$ edge flips and $2 t+1$ point moves, assuming the path does not cross through any vertices.

Proof. Suppose that $p$ is joined by edges to vertices $v_{1}, v_{2}$ and $v_{3}$. Without loss of generality, let edge $v_{2} v_{3}$ intersect the path that $p$ must follow, and let this path continue into triangle $v_{2} v_{3} v_{4}$, as shown in Figure 1.

Clearly $p$ can be moved anywhere within triangle $v_{1} v_{2} v_{3}$ without the need of any edge flips. Then it can be moved along its path, as close to edge $v_{2} v_{3}$ as necessary, so that the quadrilateral $p v_{2} v_{3} v_{4}$ becomes convex. This allows edge $v_{2} v_{3}$ to be flipped into edge $p v_{4}$. Now $p$ may continue along its path. As soon as it enters $v_{2} v_{3} v_{4}$, edge $p v_{1}$ may be flipped into $v_{2} v_{3}$. Now, with two edge flips and two point moves, $p$ has crossed through the first edge intersecting its path, and still has degree 3. By the same argument, $p$ may traverse its entire path with two edge flips and two point moves for each intersecting edge. One additional point move is required in the last triangle. Note that only three edges in the original and final triangulations will be different.

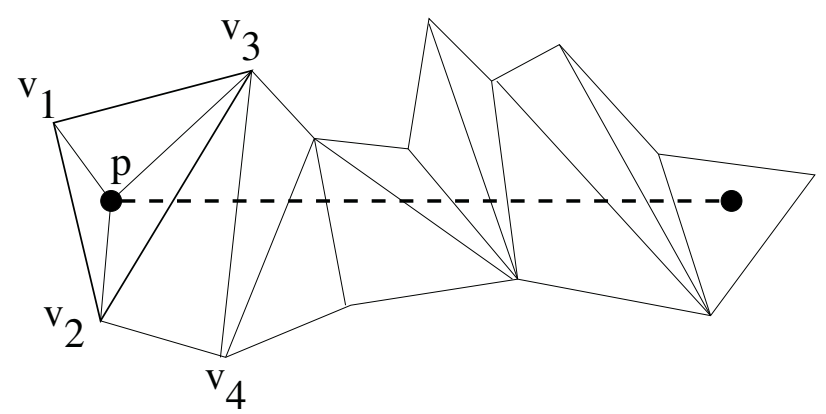

Fig. 1. A vertex $p$ and a straight path that it must move along (dashed). $p$ can pass through any edge with two edge flips.

\footnotetext{
${ }^{2}$ A triangle, defined by three consecutive vertices of a polygon, is an ear if it is empty
} and the vertices form a convex angle. The second vertex is the tip of the ear. 
Lemmata 3 and 4 imply the following result:

Lemma 5. Given a near-triangulation $T$, any vertex in the interior of the convex hull of the vertices of $T$ with degree $d$ can be moved to a new position in the triangulation along a path crossing $t$ edges, using $O(d+t)$ edge flips and point moves.

Lemma 6. An edge can be constructed between a convex hull vertex and any other vertex in a triangulation using $O(n)$ edge flips, with the aid of one moving point that is moved $O(n)$ times.

Proof. Let $v_{1}$ be the hull vertex. First suppose that the second vertex is an interior point. Then it will play the role of the moving point, and we will label it $p$. We can move $p$ directly towards $v_{1}$, until it is located within a triangle that has $v_{1}$ as a vertex. Now $v_{1}$ and $p$ must be joined with an edge. Next we move $p$ back along the same line to its original position, always maintaining edge $v_{1} p$. To do this, we consider the set of triangles that intersect $p$ 's path, as in Lemma 4. The point $p$ can always enter a triangle intersecting the path back to its original location. The difference is that once it has crossed an intersecting edge, we do not restore the edge. This means that $p$ will accumulate edge degree. An issue that needs to be taken care of is that of maintaining a triangulation when $p$ is about to lose visibility to another vertex. This occurs when one of its incident edges is about to overlap with another edge in the triangulation, as shown in Figure 2 .

Suppose that edge $p v_{3}$ is about to overlap with edge $v_{3} v_{4}$. Vertices $v_{3}$ and $v_{4}$ cannot be on opposite sides of the remaining path that $p$ must traverse, otherwise $v_{3} v_{4}$ may be flipped. The point $p$ must share an edge with $v_{4}$ in this configuration. Points $p$ and $v_{3}$ are also part of another triangle, along with some vertex $v^{*}$ which may be anywhere on the path from $v_{1}$ to $v_{3}$. These two triangles must form a convex quadrilateral $p v^{*} v_{3} v_{4}$, otherwise $p$ would have already lost visibility to $v^{*}$. Thus $p v_{3}$ may be flipped into $v_{4} v^{*}$, which means that $v_{3}$ is removed from the polygon that intersects $p$ 's path. The result is that when $p$ reaches its original position, it leaves a $\operatorname{fan}^{3}$ behind it, which includes edge $v_{1} p$.

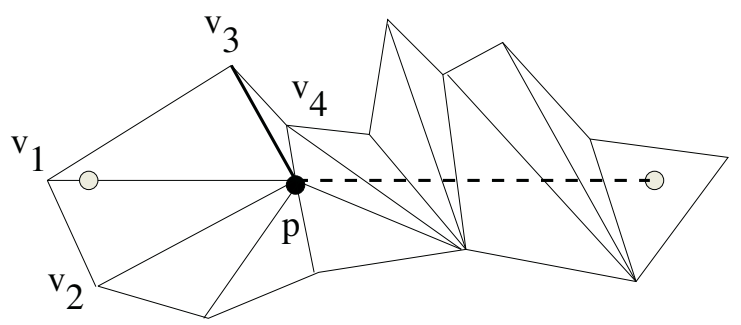

Fig. 2. Maintaining a triangulation while extending edge $v_{1} p: p$ has moved from a position close to $v_{1}$ (shown white), and still has to traverse the dashed segment to its original position. Edge $p v_{3}$ causes a problem if $p$ is to continue.

\footnotetext{
${ }^{3}$ A fan is a star-shaped polygon with a vertex as its kernel.
} 
Overall one edge flip is used when $p$ enters a new triangle, and at most one flip is used for every edge that attaches to $p$.

If both vertices of the edge that we wish to construct are on the hull, then we can take any point $p$ within the hull and move it close to $v_{1}$ and onto the segment between the two hull vertices. $p$ can then move along this segment to the second hull vertex until it is connected to both. At this moment, $p$ may be perturbed so that the three vertices form a triangle. This triangle might contain other edges incident to $p$. Lemma 2 implies that these edges may be removed so that the desired edge can be constructed with $O(n)$ edge flips.

\subsection{Triangulations}

With the basic building blocks in place, we now prove one of our main results.

Theorem 1. With $O(n \log n)$ edge flips and point moves, we can transform any geometric triangulation on $n$ points to any other geometric triangulation on $n$ possibly different points.

Proof. We transform one triangulation to another via a canonical configuration. As shown in Figure 3, the interior vertices form a backbone (i.e. their induced subgraph is a path). The top of the backbone is joined to the topmost hull vertex $v_{1}$, and all interior vertices are joined to the other two hull vertices, $v_{L}$ and $v_{R}$.

The canonical configuration is constructed in a divide-and-conquer manner. We perform a radial sweep from $v_{1}$, to find the median vertex interior to the convex hull, $v_{M}$. After constructing edge $v_{1} v_{M}$ we move $v_{M}$ directly away from $v_{1}$ towards the base $v_{L} v_{R}$, maintaining $v_{1} v_{M}$ until triangle $v_{M} v_{L} v_{R}$ contains no interior points. By Lemma 6, we use $O(n)$ operations to accomplish this. Now, we transform $v_{1} v_{M} v_{L}$ and $v_{1} v_{M} v_{R}$ into backbone configurations by induction since they are smaller instances of the same problem. The resulting configuration is shown in Figure 4.

We now show that the two sides may be merged using $O(n)$ operations. As shown in Figure 5a, we first move the lowest vertex of a backbone into a position that is close to the base and is along the extension of edge $v_{1} v_{M}$. This requires one edge flip. The vertices on the left/right backbones are processed in

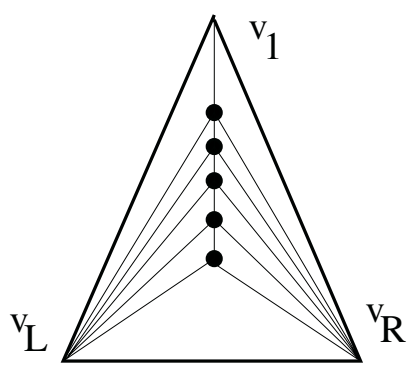

Fig. 3. The canonical configuration used for triangulations. 


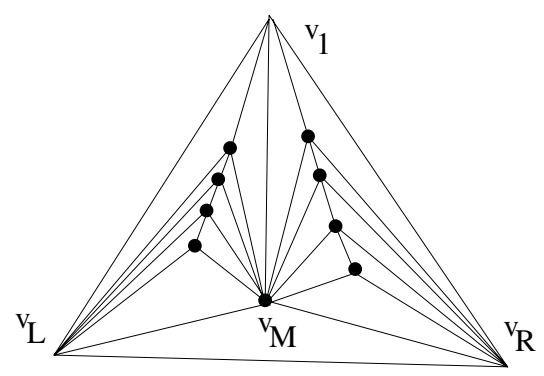

Fig. 4. The configuration of a triangulation prior to merging the backbones on each side of the median vertex $v_{M}$.
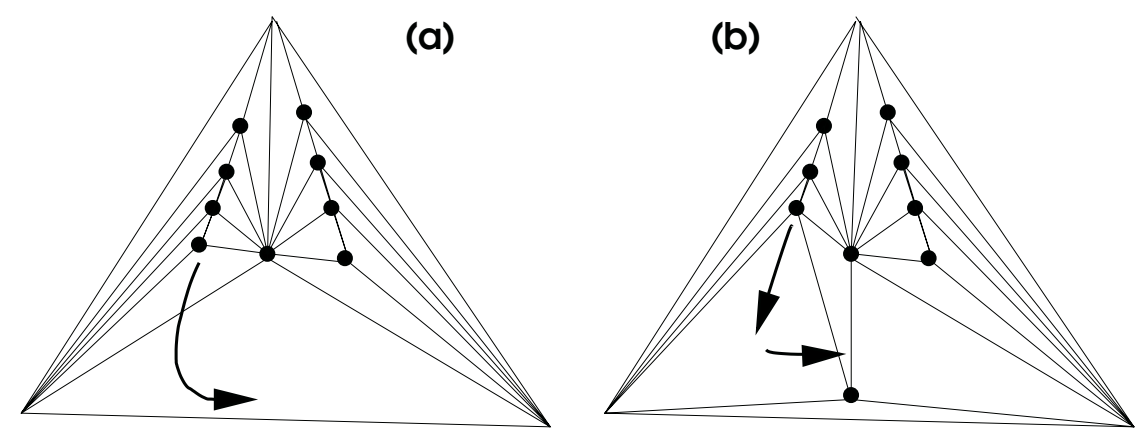

Fig. 5. Merging two backbones into one.

ascending order, and are always moved just above the previous processed vertex, as shown in Figure 5b. Each vertex will require two point moves and one edge flip. Thus $v_{1} v_{L} v_{R}$ is reconfigured into canonical form, and by a simple recurrence the number of edge flips and point moves used is $O(n \log n)$. It is trivial to move a canonical triangulation to specific coordinates using $n$ point moves. Thus the transformation between any two triangulations may be completed.

\subsection{Near-Triangulations}

If the initial graph is a near-triangulation, Theorem 1 does not directly apply. Some care must be taken to handle a non-triangular outer face. Details are given in the proof of the following theorem:

Theorem 2. With $O(n \log n)$ edge flips and point moves, we can transform any geometric near-triangulation on $n$ points to any other geometric near-triangulation on $n$ possibly different points.

Proof. As in the case with triangulations, we transform one near-triangulation to another via a canonical configuration. In the primary canonical configuration, shown in Figure 6, one chosen hull vertex $\left(v_{1}\right)$ is joined by chords to all other 


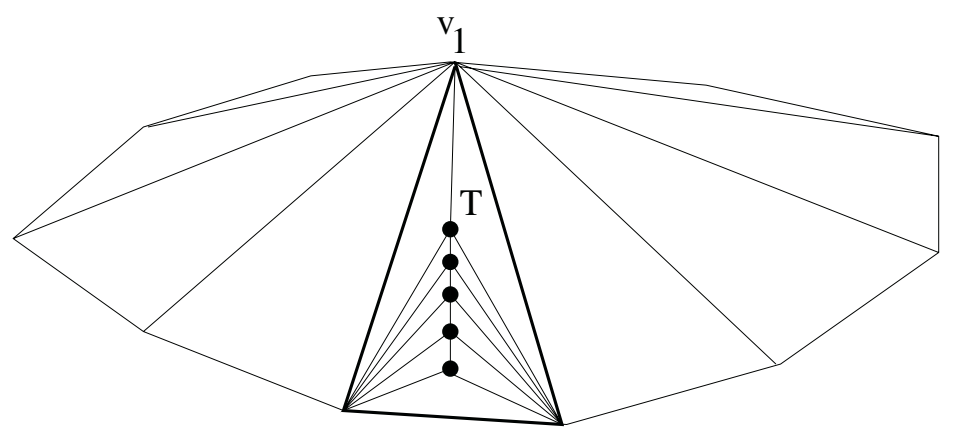

Fig. 6. The primary canonical configuration used for near-triangulations.

hull vertices. Thus $v_{1}$ is in the kernel of a convex fan. Every triangle of the fan, except for one, is empty. All interior vertices, located in the non-empty triangle $T$, are in the canonical configuration of a triangulation.

We first construct all edges of the top-level fan configuration, leaving interior vertices in their original positions. Then within each triangle of the fan, we rearrange the interior vertices into a canonical triangulation. Finally, we merge all triangles of the fan, so that all interior points move to a single triangle and are in canonical form.

To construct the fan chords, we always divide the problem into two roughly equal parts. We begin by constructing two chords as follows: perform a radial sweep from $v_{1}$ to successive hull vertices $v_{i}\{2 \leq i \leq n-1\}$, always keeping fewer than $\frac{n}{2}$ vertices in the swept region. Let $v_{j}$ be the last hull vertex for which this holds. Construct chords $v_{1} v_{j}$ and $v_{1} v_{j+1}$. The unswept region not including triangle $v_{1} v_{j} v_{j+1}$ contains fewer than $\frac{n}{2}$ vertices. The swept region contains fewer than $\frac{n}{2}$ vertices. Triangle $v_{1} v_{j} v_{j+1}$ may contain an arbitrary number of vertices, but this is not a sub-problem (we will not look at this region again during the construction of the fan). Now we can continue a new sweep on each side of $v_{1} v_{j} v_{j+1}$. Construction of the two chords could take $O(n)$ edge flips and point moves, as described in Lemma 6. However the even split of the sub-problems ensures that the total number of operations is $O(n \log n)$.

Each fan triangle $v_{1} v_{i} v_{i+1}$, containing $k_{i}$ interior points, can be reconfigured into a backbone structure with $O\left(k_{i} \log k_{i}\right)$ operations, by Theorem 1 . Thus the total number of edge flips and point moves used to reconfigure all triangles of the fan into backbone structures is $O(n \log n)$.

Now we are left only with the task of merging the fan triangles so that only one of them will contain all interior points. We can add $k_{i}$ interior points of a canonical triangulation to an adjacent canonical triangulation using $O\left(k_{i}\right)$ edge flips and point moves. The $k_{i}$ points are processed in descending order and are always added to the top of the adjacent triangulation, as shown in Figure 7.

Thus we obtain one triangle in canonical form next to an empty triangle. It is just as easy to merge two canonical triangles separated by an empty triangle. If we encounter two or more adjacent empty fan triangles, we may use Lemma 1 


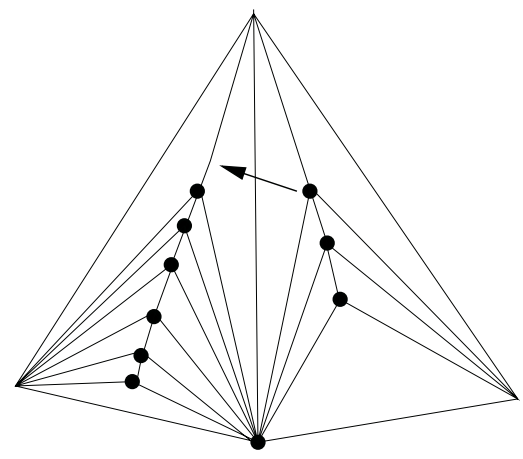

Fig. 7. Merging two adjacent fan triangles.

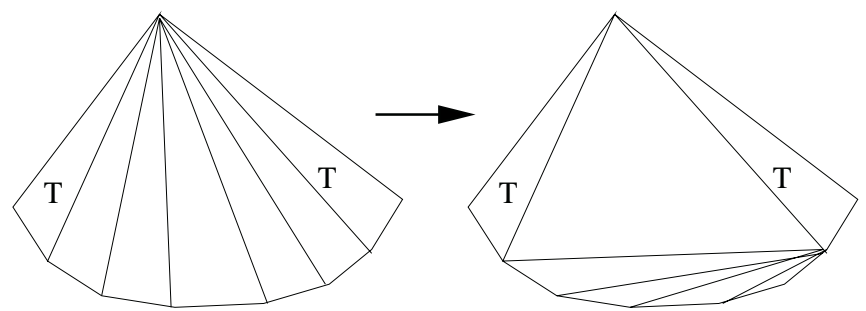

Fig. 8. Handling multiple adjacent empty fan triangles. Triangles marked $(\mathrm{T})$ contain triangulations.

to reconfigure them so that they will not affect the fan-merging process (see Figure 8). By the above arguments, once we select the triangle that is to finally contain all of the interior points (the median triangle is a good choice), we can iteratively merge its neighboring triangles onto it using a total of $O(n)$ edge flips and point moves.

Finally we are left with a single triangle containing all interior points in canonical form. On either side, we may have an arbitrary triangulation (resulting from handling multiple adjacent empty fan triangles), but the vertices will be in convex position. By Lemma 1 they may be moved to our desired configuration using $O(n)$ edge flips.

We must still show that this primary canonical configuration can be moved to specific coordinates. This can be done with $O(n)$ point moves, though space restrictions prevent us from going into any detail.

\subsection{Remarks}

Our algorithms also work for labeled triangulations, with minor care needed. Constructing the canonical configuration resembles merge-sort.

If two triangulations have the same point set, the problem is no easier than the general problem. Suppose that there exists an algorithm that can transform a triangulation $T_{1}$ on a given $n$-point set to a triangulation $T_{2}$ on the same point 


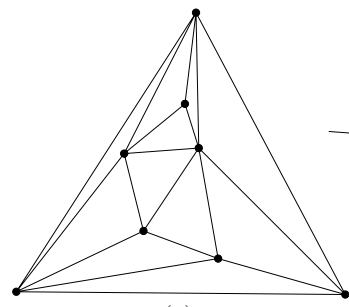

(a)

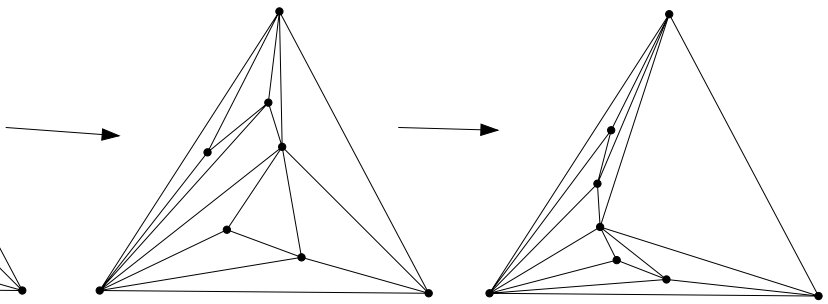

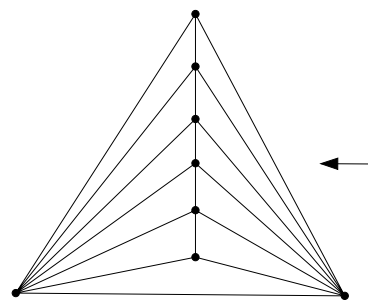

(e) (c)

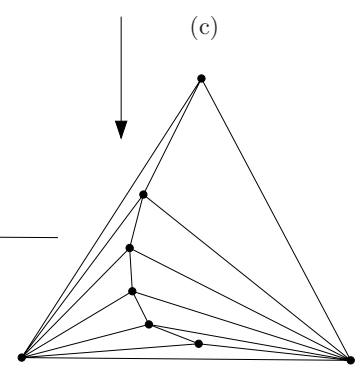

(d)

Fig. 9. Problem on fixed point set is not easier.

set using $F_{n}=o(n \log n)$ edge flips and point moves. Then this algorithm can be used to transform a triangulation on one point set to any other triangulation on a possibly different point set with $F_{n}+O(n)$ edge flips and point moves. This argument is summarized in Figure 9. Let Figure 9a be the input triangulation. With $F_{n}$ flips and moves, move to the triangulation in Figure $9 \mathrm{~b}$ where every vertex is adjacent to the lower left vertex $v_{\ell}$ of the outer face.

Now consider the triangulated polygon, $P$, that consists of edges not adjacent to $v_{\ell}$. Notice that if we perform a radial sweep from $v_{\ell}$, the boundary of $P$ is monotonic. At least two of the triangles in $P$ are disjoint ears, which means there must exist an ear tip that is an interior vertex and is also joined to $v_{\ell}$ by an edge in the original triangulation. We may move this point directly towards $v_{\ell}$ and cut the ear from $P$. This still leaves a monotone polygon $P^{\prime}$. By continuously locating such ears, and moving them to a predefined convex position, we can obtain the configuration illustrated in Figure 9c. The monotonicity of $P$ (and its descendants) and the convexity of the final configuration of interior points guarantee that no edge crossings will occur. This process requires a linear number of point moves.

Next, by Lemma 1, we can use $O(n)$ edge flips to obtain the triangulation where the lower right vertex of the outer face is adjacent to every vertex, as illustrated in Figure 9d. From here, it is trivial to move to the canonical configuration.

We conclude with the following:

Theorem 3. If an algorithm exists that can reconfigure between any two geometric triangulations of the same point set with $o(n \log n)$ edge flips and point 
moves, then we can also transform any geometric triangulation on $n$ points to any other geometric triangulation on $n$ different points with o $(n \log n)$ flips and moves.

It is tempting to try to find a fast algorithm that will construct a monotone path, as illustrated in the transition from Figure 9a to Figure 9b. Consider the polygon that is the union of all triangles incident to the lower left vertex of Figure 9b. By continuously cutting ears of this polygon, we may get to a triangulation that is similar to that of Figure 9a, using $O(n)$ edge flips. The similarity is that all neighbors of the lower left vertex will be in convex position. However, we have little control over the resulting positions of the remaining edges if we use only $O(n)$ operations. It is possible to create triangulations for which the reversal of this ear-cutting technique is not possible. In fact, Figure $9 \mathrm{c}$ serves as an example, if we add a few more vertices inside the large triangle. In this figure none of the edges directly visible from the lower left vertex can be flipped, so there is no obvious way to achieve a monotone path with fewer than $O(n \log n)$ operations.

We finally consider the following more powerful point move as an alternative to the point move studied so far. In this more powerful point move, we can delete an interior vertex of degree three (and all its incident edges), and create a new vertex of degree three inside another triangle of the triangulation. With this type of move we can reconfigure triangulations using $O(n)$ operations. We simply select a triangle incident to a hull edge and create a backbone inside. This is done by continuously selecting a vertex of constant degree from outside the triangle, reducing its degree to three, and moving it to the lower end of the backbone.

\section{References}

1. Abellanas, M., Bose, P., Garcia, A., Hurtado, F., Ramos, P., Rivera-Campo, E., Tejel, J.: On local transformations in plane geometric graphs embedded on small grids. In Proceedings of the International Workshop on Computational Geometry and Applications (CGA) 2 (2004) 22-31

2. Bose, P., Czyzowicz, J., Gao, Z., Morin, P., Wood, D.: Parallel diagonal flips in plane triangulations. Tech. Rep. TR-2003-05, School of Computer Science, Carleton University, Ottawa, Canada (2003)

3. Brunet, R., Nakamoto, A., Negami, S.: Diagonal flips of triangulations on closed surfaces preserving specified properties. J. Combin. Theory Ser. B 68(2) (1996) 295-309

4. Cortés, C., Grima, C., Marquez, A., Nakamoto, A.: Diagonal flips in outertriangulations on closed surfaces. Discrete Math. 254(1-3) (2002) 63-74

5. Cortés, C., Nakamoto, A.: Diagonal flips in outer-torus triangulations. Discrete Math. 216(1-3) (2000) 71-83

6. Galtier, J., Hurtado, F., Noy, M., Pérennes, S., Urrutia, J.: Simultaneous edge flipping in triangulations. Internat. J. Comput. Geom. Appl. 13(2) (2003) 113133

7. Gao, Z., Urrutia, J., Wang, J.: Diagonal flips in labelled planar triangulations. Graphs Combin. 17(4) (2001) 647-657 
8. Hurtado F., Noy, M.: Graph of triangulations of a convex polygon and tree of triangulations. Comput. Geom. 13(3) (1999) 179-188

9. Hurtado, F., Noy, M., Urrutia, J. Flipping edges in triangulations. Discrete Comput. Geom. 22(3) (1999) 333-346

10. Komuro, H.: The diagonal flips of triangulations on the sphere. Yokohama Math. J. 44(2) (1997) 115-122

11. Komuro, H., Nakamoto, A., Negami, S.: Diagonal flips in triangulations on closed surfaces with minimum degree at least 4. J. Combin. Theory Ser. B 76(1) (1999) 68-92

12. Lawson, C.: Software for $c_{1}$ surface interpolation. In J. Rice, ed., Mathematical Software III, pp. 161-194, Academic Press, New York (1977)

13. Meisters, G.: Polygons have ears. American Mathematical Monthly 82 (1975) 648651

14. Nakamoto, A., Negami, S.: Diagonal flips in graphs on closed surfaces with specified face size distributions. Yokohama Math. J. 49(2) (2002) 171-180

15. Negami, S.: Diagonal flips in triangulations of surfaces. Discrete Math.135(1-3) (1994) 225-232

16. Negami, S.: Diagonal flips in triangulations on closed surfaces, estimating upper bounds. Yokohama Math. J. 45(2) (1998) 113-124

17. Negami, S.: Diagonal flips of triangulations on surfaces, a survey. Yokohama Math. J. 47 (1999) 1-40

18. Negami, S., Nakamoto, A.: Diagonal transformations of graphs on closed surfaces. Sci. Rep. Yokohama Nat. Univ. Sect. I Math. Phys. Chem. 40 (1993) 71-97

19. Wagner, K.: Bemerkung zum Vierfarbenproblem. Jber. Deutsch. Math.-Verein. 46 (1936) 26-32

20. Watanabe, T., Negami, S.: Diagonal flips in pseudo-triangulations on closed surfaces without loops. Yokohama Math. J. 47 (1999) 213-223 